\title{
THE SUN AS A CALIBRATION SIGNAL SOURCE FOR L- AND S-BAND TELEMETRY
}

\author{
W. R. HEDEMAN \\ Manager of Telemetry and Instrumentation Section \\ Aerospace Corporation \\ San Bernardino, California
}

Summary One of the major problems confronting a telemetry receiving station is that of self calibration, particularly an end-to-end calibration, on a frequent and routine basis. For this purpose an external signal source is needed, preferably one in the far field of the antenna. The sun is such a source for L- and S-band systems--its usefulness depends on knowledge of its emission at the time it is used, since it is a variable source.

Examined here are the characteristics of the sun as a source of electromagnetic energy in the 10 centimeter region, and the methods by which it could be used to determine receiving system noise temperature. Limitations of the methods are also described.

Discussion The most rigorous calibration of a telemetry system requires a far field source free of multipath transmission phenomena producing a known field intensity at the receiving antenna aperture. The source should be modulated to simulate an actual operation, and the receiving system should be in the operational configuration. Performance of the system should be measured at the output terminals of the system, usually a playback from a magnetic tape. (Ref 1.)

For a 24-ft diameter receiving antenna at $2250 \mathrm{MHz}$ a source would have to be at least $2500 \mathrm{ft}$ distant to be in the far field. At this distance, it would have to be at least $60 \mathrm{ft}$ above the local horizon to place the first antenna minimum above the horizon. These are formidable and expensive constraints, particularly for mobile stations.

An alternative to system calibration in one step is to calibrate in two steps. This is possible since the noise performance of the system is principally determined in the radio frequency circuits. The principal variables are antenna efficiency, transmission line losses, and preamplifier noise temperature, if one assumes that local electromagnetic interference has been controlled and reduced to a negligible value. The wide band characteristics of the circuit elements involved make them generally insensitive to signal characteristics. (One should question the intermodulation characteristics of the 
preamplifier if numbers of carriers are used in a particular operation, but this can be resolved independently of a noise performance test. )

In addition, changes in performance of radio frequency circuit elements usually occur slowly. The principal effects are due to local weather (temperature, precipitation, humidity) and constituents of the local atmosphere (corrosive gases and dust). Calibration of this portion of the system needs to be performed periodically, but not necessarily prior to each operation. Calibration should certainly be accomplished whenever the system is maintained, repaired or overhauled.

Provision should also be made for determining system performance in an operational configuration as recommended by Nichols. (Ref. 1. ) For this test the signal can be injected at the pre or postamplifier input terminals, with the former to be preferred, via a directional coupler.

Alternate candidates for the far field signal source for system noise calibration have been considered. The obvious candidate is a calibration satellite in a polar orbit, which would be visible two to four times each day from every telemetry station on the surface of the earth. Scheduling and on-off control by command would be required since it does not seem to be reasonable to provide battery reserve for continuous operation. An ephemeris would be needed to calculate pointing information--computer generated angles for each telemetry station requiring calibration might be desirable. Once a calibration satellite became standard, such services should not be denied for periods in excess of one week; i.e., the time between the demise of one and the appearance of its replacement in orbit should not exceed one week, and should preferably be considerably less.

The sun, and other stars, represent available sources of wideband energy in the L- and S bands. The sun is perhaps the most applicable, since the level of emission, even during quiet periods appears to be more than adequate to calibrate receiving antennas as small as $6 \mathrm{ft}$ in diameter. The angular size of the source is such that antennas as large as $60 \mathrm{ft}$ in diameter can use the source. Between these two limits of size one finds the great majority of telemetry receiving antennas. The few larger antennas have adequate aperture to use radio stars other than the sun, as for instance Cassiopeia A, for calibration.

The sun, however, is a variable source, and the accuracy of measurements depend upon knowledge of the sun's activity at the time it is used. Solar radio astronomers around the world have the sun under continuous observation in the decimeter region, and the accuracy of the data is limited only by the accuracy with which each solar observatory is calibrated. Calibration errors, in general, are less than those which would be associated with a calibration satellite when one considers the uncertainties of power output, antenna patterns, and vehicle attitude. 
Characteristics of Solar Emission in the 10 Centimeter Region Solar emission in the decimeter band consists of three basic components: (1) radiation from the "quiet" sun, (2) a slowly varying cornponent which changes with sunspot number, and (3) occasional radio bursts which last from several seconds to several hours. Throughout the band for all three components the spectrum is essentially continuous, i.e., featureless in the frequency domain, and is basically randomly polarized. (Hachenburg in Aarons, Ref. 2.)

Radiation from the quiet sun is considered to be the level which would be observed if there were no sunspot activity, and in the decimeter region is approximately 70 flux units during the minimum of the sunspot cycle. (One flux unit $=10^{22}$ watts $\mathrm{m}^{-2} \mathrm{~Hz}^{-1}$.) The radiation level published by solar astronomers is that which would be observed just outside of the sensible atmosphere of the earth.

The sum of quiet sun radiation and the slowly varying component is shown in Figure 1, together with sunspot number. Correlation of the variation is evident. Figure 2 shows monthly maxima and minima. The monthly minimum is a rough approximation to the quiet sun level. Hachenburg (Aarons, Ref. 2) estimates an average error of daily measurements to be \pm 4 percent based on a comparison of results from different places at nearly the same frequencies. Covington (Ref. 3) estimates "Constant errors associated with the absolute measurements of flux" to be \pm 7 percent.

Radio bursts or sudden enhancements are usually associated with solar flares--great bursts always, small bursts sometimes. Figure 3 shows the distribution of burst intensities observed at Ottawa by Covington (Ref. 3) over a period of years.

The sun is useful, then, as a source of calibrating signals. The accuracy with which it can be used depends on knowledge of the level of activity at the time a calibration is made. The sun is under constant observation by solar observatories around the world. If four or five of these provided continuous coverage (each could observe for at least six hours each day) of the sun in the L- and S-telemetry bands, and were connected into essentially real time communications network available to telemetry receiving stations, knowledge of total solar flux would be as good as the calibration of the active observatory at any time. To simplify the system somewhat, receiving stations might calibrate on the hour sometime between 0900 and 1500 hours local time. Only hourly observatory readings would then be needed on the communications network.

Twice daily readings of total solar flux at $10.7 \mathrm{~cm}$ are made at Ottawa by the National Research Council, and are made available on a national teletype service by the Environmental Sciences Service Administration from Boulder, Colorado. These daily readings contain the quiet sun component and the slowly varying component. If one knew that solar flare activity was not in process at the time of a calibration, this daily 
total flux value could be used. An examination of the daily readings for the last half of 1957 (Ref. 4), during the sunspot maximum, shows a maximum change from one day to the next of approximately 10 percent.

Due to broad band characteristics of solar radio noise in the decimeter region some authors indicate that interpolation between spot frequencies may be used to determine solar activity at other frequencies in the band. However, the errors associated with this approximation seem to be uncertain. The better procedure would be to make observations in the assigned L- and S-telemetry bands.

Calculation of System Noise Temperature If a highly directive pencil beam is pointed at the center of a radio source which is a circular disk of angular diameter $\theta_{\mathrm{d}}$ with uniform brightness temperature $\mathrm{T}_{\mathrm{d}}$, and the background brightness is zero, the observed antenna temperature $\mathrm{T}_{\mathrm{A}}$ due to the radio source is:

$$
\mathrm{T}_{\mathrm{A}}=\eta_{\mathrm{r}} \frac{\iint_{\text {source }} \mathrm{T}_{\mathrm{d}} \mathrm{f}(\theta, \phi) \mathrm{d} \Omega}{\iint_{4 \pi} \mathrm{f}(\theta, \phi) \mathrm{d} \Omega}
$$

where $\quad \eta_{\mathrm{r}}=$ the radiation efficiency of the antenna

$\mathrm{f}(\theta, \phi)=$ the radiation power pattern of the antenna normalized with respect to the maximum radiation intensity

$$
\begin{aligned}
\mathrm{d} \Omega & =\text { an element of solid angle } \\
& =\sin \theta \mathrm{d} \theta \mathrm{d} \phi .
\end{aligned}
$$

The antenna solid angle $\Omega_{\mathrm{A}}$ is given by

$$
\Omega_{\mathrm{A}}=\iint_{4_{\pi}} \mathrm{f}(\theta, \phi) \mathrm{d} \Omega
$$

It is well known in antenna theory (Ref. 5) that

$$
\Omega_{\mathrm{A}}=\eta_{\mathrm{r}} \frac{\lambda^{2}}{\mathrm{~A}_{\mathrm{em}}}
$$

where $\quad \lambda=$ wave length

$\mathrm{A}_{\mathrm{em}}=$ the maximum effective aperture of the antenna. 
If the solid angle subtended by the disk is $\Omega_{\mathrm{d}}$ then Equations (1) and (2) become

$$
{ }_{\mathrm{T}_{\mathrm{A}}}=\eta_{\mathrm{r}} \mathrm{T}_{\mathrm{d}} \frac{\Omega \mathrm{d}}{\Omega_{\mathrm{d}}} \frac{\iint_{\text {source }} \mathrm{f}(\theta, \phi) \mathrm{d} \Omega}{\Omega_{\mathrm{A}}}
$$

or

$$
\mathrm{T}_{\mathrm{d}}=\frac{\mathrm{T}_{\mathrm{A}}}{\eta_{\mathrm{r}}} \frac{\Omega \mathrm{A}}{\Omega_{\mathrm{d}}} \quad\left\{\frac{\Omega_{\mathrm{d}}}{\iint_{\text {source }} \mathrm{f}(\theta, \phi) \mathrm{d} \Omega}\right\}
$$

The radiation from the disk is randomly polarized, and the antenna is assumed to be a single polarization. The flux density of the radio source is:

$$
\begin{array}{rl}
F & =\iint_{\substack{\text { source } \\
2 \mathrm{KTd}}} \frac{2 \mathrm{KT}_{\mathrm{d}}}{\lambda^{2}} \mathrm{~d} \Omega \\
\lambda^{2} & \mathrm{~d}
\end{array}
$$

where $\quad \mathrm{K}=$ Boltzmann's constant.

Substituting the value of $\mathrm{T}_{\mathrm{d}}$ from Equation (5) in Equation (7), using $\Omega_{\mathrm{A}}$ from Equation (3)

$$
F=\frac{2 \mathrm{KT}_{\mathrm{A}}}{\mathrm{A}_{\mathrm{em}}}\left\{\frac{\Omega_{\mathrm{d}}}{\iint_{\text {source }} \mathrm{f}(\theta, \phi) \mathrm{d} \Omega}\right\}
$$

A correction factor is now defined as

$$
\mathrm{L}=\frac{\Omega_{\mathrm{d}}}{\iint_{\text {source }} \mathrm{f}(\theta, \phi) \mathrm{d} \Omega},
$$


and Equations (5) and (8) become

$$
\begin{aligned}
\mathrm{T}_{\mathrm{d}} & =\frac{\mathrm{T}_{\mathrm{A}}}{\eta_{\mathrm{r}}} \frac{\Omega_{\mathrm{A}}}{\Omega_{\mathrm{d}}} \mathrm{L} \\
\mathrm{F} & =\frac{2 \mathrm{~K} \mathrm{~T}_{\mathrm{A}}}{\mathrm{A}_{\mathrm{em}}} \mathrm{L} .
\end{aligned}
$$

The correction factor becomes unity when the source is so small that $f(\theta, \phi)$ is unity over the disk. When this is not the case, the surface integral in Equation (9) must be evaluated. Ko (Ref. 6) has performed this calculation for three representative power patterns. Table I summarizes his results.

The distribution of temperature over the solar disk is not uniform at 10 centimeters wavelength. Figure 4 shows calculated values of brightness temperature as a function of distance from the center of the optical disk. It is seen that the radio disk is slightly larger than the optical disk, at 10 centimeters, and that some brightening occurs near the edge of the optical disk (limb brightening). Though it is not shown, limb brightening in the polar regions is different from that in the equatorial regions.

When the temperature distribution is not uniform over the surface, the more exact form of Equation (1) is:

$$
\mathrm{T}_{\mathrm{A}}=\eta_{\mathrm{r}} \frac{\iint_{\text {source }} \mathrm{T}_{\mathrm{d}}(\theta, \phi) \mathrm{f}(\theta, \phi) \mathrm{d} \Omega}{\iint_{4 \pi} \mathrm{f}(\theta, \phi) \mathrm{d} \Omega}
$$

When the temperature distribution and the power pattern are known, Equation (12) can be evaluated for a particular case.

The observed antenna temperature, $\mathrm{T}_{\mathrm{A}}$, is that which would be seen by the actual antenna if it was located just outside of the sensible atmosphere. If the actual antenna is pointed away from the sun, assuming side lobe levels do not contribute materially to system noise temperature, this temperature is:

$$
\mathrm{T}_{\mathrm{Sl}}=\mathrm{T}_{\mathrm{Cl}}+\mathrm{T}_{\mathrm{atl}}+\frac{\mathrm{T}_{\mathrm{gl}}}{\epsilon_{\mathrm{at} 1}}+\frac{\mathrm{T}_{\mathrm{r}}}{\epsilon_{\mathrm{at} 1}}
$$


If the antenna is now pointed toward the sun, the system noise temperature is:

$$
\mathrm{T}_{\mathrm{S} 2}=\frac{\mathrm{T}_{\mathrm{A}}}{\eta_{\mathrm{r}}}+\mathrm{T}_{\mathrm{C} 2}+\mathrm{T}_{\mathrm{at2}}+\frac{\mathrm{T}_{\mathrm{g} 2}}{\epsilon_{\mathrm{at2}}}+\frac{\mathrm{T}_{\mathrm{r}}}{\epsilon_{\text {at2 }}}
$$

where $\quad \mathrm{T}_{\mathrm{C}}=$ cosmic noise temperature

$\mathrm{T}_{\mathrm{at}}=$ atmospheric noise temperature

$\mathrm{T}_{\mathrm{g}}=$ environmental noise temperature, principally through side and back lobes from the earth

$\mathrm{T}_{\mathrm{r}}=$ noise temperature contribution from the receiver, referred to the antenna input terminals

$\epsilon_{\mathrm{at}}=$ atmospheric transmission efficiency,

and the subscripts 1 and 2 refer to a main lobe direction away from the sun and toward the sun, respectively.

If we assume that cosmic, atmospheric, and environmental noise do not change between the two observations, then

$$
\mathrm{T}_{\mathrm{S} 2}=\frac{\mathrm{T}_{\mathrm{A}}}{\eta_{\mathrm{r}}}+\mathrm{T}_{\mathrm{S} 1}
$$

if $\mathrm{P}_{2}$ and $\mathrm{P}_{1}$ are the predetection power outputs from the receiver corresponding to the two pointing directions, and the available system gain does not change between the two observations, then

$$
\frac{\mathrm{P}_{2}}{\mathrm{P}_{1}}=\frac{\mathrm{T}_{\mathrm{A}} / \eta \mathrm{r}+\mathrm{T}_{\mathrm{S} 1}}{\mathrm{~T}_{\mathrm{S} 1}}
$$

or

$$
\mathrm{T}_{\mathrm{S} 1}=\frac{\mathrm{T}_{\mathrm{A}} / \eta_{\mathrm{r}}}{\mathrm{P}_{2} / \mathrm{P}_{1}-1}
$$

$\mathrm{T}_{\mathrm{A}}$ is calculated from Equation (11) and Table I, or more exactly from Equation (12), if necessary, and $\mathrm{T}_{\mathrm{S} 1}$ can be determined.

If all observations are made with pointing angles of less than 45 deg away from the zenith the principal noise contribution to $\mathrm{T}_{\mathrm{S}}$ from sources other than the receiver will be environmental noise. The sum of cosmic, atmospheric, and environmental clear sky noise temperatures for simple parabolic antennas at S-band has been estimated to be between $40^{\circ} \mathrm{K}$ and $50^{\circ} \mathrm{K}$ for a receiving station located on land, and perhaps as low as $100 \mathrm{~K}$ for a 
receiving station located on water, or above a reflecting ground plane (Refs. 8 and 9). At angles near zenith atmospheric attenuation at S-band is negligible (Ref. 10). Therefore, the principal noise factor in $\mathrm{T}_{\mathrm{S} 1}$ for the majority of telemetry stations will be the receiver contribution.

In the day to day calibration of telemetry receiving stations, one is interested principally in any change in calibration, not necessarily in an absolute calibration. It will usually be sufficient to calculate $\mathrm{T}_{\mathrm{S} 1}$ as in Equation (17), rather than attempting to calculate receiver noise temperature or noise figure, though this may be done if detailed receiving system characteristics are available.

From the work of Ko (Ref. 6) the antenna correction factor, L, may be assumed to be unity for antenna (maximum) gain less than $40 \mathrm{db}$, and a correction factor applied for antennas with gains not exceeding $50 \mathrm{db}$. For antennas with gains in excess of $50 \mathrm{db}$, the actual antenna power function may be convolved with the actual temperature brightness distribution over the sun to obtain antenna temperature. However, for these larger antennas it may be possible to use weaker sources such as the moon or radio stars. Castelli (Ref. 11) calibrated an 84-ft dish against Cassiopeia A, and using Ko's correction factor obtained solar flux measurements which agreed reasonably well with measurements made by Covington at Ottawa.

For smaller antennas and higher noise figure receivers, the power ratio $\mathrm{P}_{2} / \mathrm{P}_{1}$ (Equation 16) will approach unity, and the calculation of system noise temperature will become inaccurate. At $10 \mathrm{~cm}$ the sunspot minimum quiet sun noise temperature is approximately $250^{\circ} \mathrm{K}$ per square meter of effective aperture for a single polarization. If

\section{$250 \mathrm{~A}_{\mathrm{em}}>\mathrm{T}_{\mathrm{Sl}}$}

the percentage error in $\mathrm{T}_{\mathrm{S} 1}$ due to power measurements will be less than twice the percentage error in $\mathrm{P}_{2} / \mathrm{P}_{1}$ during the sunspot minimum, and even less as the sunspot cycle proceeds. The smallest antenna which can be calibrated successfully using this method will depend upon the receiver noise figure, the gain stability of the receiver between the two measurements, and the constancy of noise contributions other than those due to the sun between the two measurements. It is estimated that, when using room temperature parametric amplifiers, effective apertures of somewhat less than one square meter can be calibrated.

\section{The Effect of the Sun on Mission Planning and Antenna Design}

For larger apertures and low noise figure receivers solar noise can be very important, and could seriously affect daytime system performance. Several currently planned antennas will be $80 \mathrm{ft}$ in diameter, with a receiver noise figure of $1.6 \mathrm{db}$. The quiet sun main lobe 
noise temperature would be $50,000^{\circ} \mathrm{K}$, and the disturbed sun could contribute $1.5 \mathrm{x}$ $10^{6 \circ} \mathrm{K}$. The receiver noise temperature (equivalent to a noise figure of $1.6 \mathrm{db}$ ) is $130 \mathrm{~K}$, and other factors might increase the system noise temperature to approximately $200^{\circ} \mathrm{K}$. To reduce the solar noise temperature to a reasonable value, say $50^{\circ} \mathrm{K}$ (equivalent to 1 $\mathrm{db}$ loss in system performance), requires $30 \mathrm{db}$ of sidelobe suppression during the quiet sun, and $45 \mathrm{db}$ of suppression during the disturbed sun. It is evident that major side lobes (less than $30 \mathrm{db}$ below the main lobe) should not transit the sun at critical times in a mission during the quiet sun. During the disturbed sun, it appears that daytime system performance could be degraded somewhat at all times, and as much as $9 \mathrm{db}$ when a minor lobe sun transit occurs.

Even modestly sized apertures should not transit the sun with the main lobe at critical times in a mission. Apertures of slightly less than one square meter, when used with a $1.6 \mathrm{db}$ preamplifier, will suffer an effective $3 \mathrm{db}$ loss in system performance if the main lobe transits the quiet sun.

It seems evident that L- and S- band telemetry antennas are much more critical with respect to side lobe suppression, because of the sun, than their VHF counterparts. Failure to provide close to optimum side lobe suppression may constrain or even inhibit daytime telemetry missions, particularly when the sun is disturbed.

Conclusions The sun provides adequate energy for the calibration of the majority of Land S-band telemetry receiving antennas, and may be considered to be a point source for receiving antenna gains of less than $50 \mathrm{db}$. The accuracy of calibration is limited only by the accuracy of calibration of solar radio observatory antennas. If solar observatory readings are made available for use via a real time, or reasonably short delay, communications link, the sun would be equal to or better than a calibration satellite in terms of accuracy of calibration and information available.

\section{References}

1. M. H. Nichols, "Requirements for a Data Quality Assurance Program", Proc. of the International Telemetry Conference, Vol. II (1966), pp. 535.

2. J. Aarons (ed), Solar System Radio Astronomy, Plenum Press, New York, New York (1965).

3. A. E. Covington, "Solar Emission at Ten Centimeter Wavelength, 1947-1960”, J. Royal Astron, Soc. Canada, 55, pp. 166 (1961).

4. S. L. Valley, Handbook of Geophysics and Space Environments, McGraw-Hill Book Company, New York, New York (1965).

5. J. D. Kraus, Antennas, McGraw-Hill Book Company, Inc., New York New York (1950). 
6. H. C. Ko, "On the Determination of the Disk Temperature and the Flux Density of a Radio Source Using High Gain Antennas", IRE Transactions on Antennas and Propagation, pp. 500-501 (September 1961).

7. J. L. Pawsey and S. F. Smerd, "Solar Radio Emission", The Sun, (G. P. Kuiper, ed), University of Chicago Press (1953).

8. R. C. Hansen, "Low Noise Antennas", Microwave Journal, pp. 1924 (June 1959).

9. J. Greene, "Antenna Noise Temperature" AIL Advertisement, Proc-. IRE, Vol. 46, pp. 2a, (May 1958).

10. J. D. Kraus, Radio Astronomy, McGraw-Hill Book Company, Inc., New York, New York (1966).

11. J. P. Castelli, Solar Flux Measurements, April 1962, and Antenna Consideration, AFCRL-62-1111, (December 1962). (ASTIA Doc. No. 298646)

TABLE I

CORRECTION FACTOR FOR ANTENNA POWER PATTERN

\begin{tabular}{|c||c|c|}
\hline $\begin{array}{c}\text { Power } \\
\text { Pattern }\end{array}$ & 1.0 & 2.0 \\
\hline \hline $\exp \left[-(\mathrm{p} \theta)^{2}\right]$ & 1.39 & 2.96 \\
\hline$\left[\frac{\sin (\mathrm{p} \sin \theta)}{\mathrm{p} \sin \theta}\right]^{2}$ & 1.38 & 3.19 \\
\hline$\left[\frac{2 \mathrm{~J}_{1}(\mathrm{p} \sin \theta)}{\mathrm{p} \sin \theta}\right]^{2}$ & 1.38 & 3.15 \\
\hline
\end{tabular}

Values of correction factor, $\mathrm{L}$, as a function of $\theta_{\mathrm{d}}$ the angular diameter of the source, $\theta_{\mathrm{H}}$ the half-power beamwidth of the antenna, and the antenna power pattern. For $\theta_{d} / \theta_{H}<1$ the expression $\mathrm{L} \cong 1+0.38\left(\theta_{\mathrm{d}} / \theta_{\mathrm{H}}\right)^{2}$ can be used for these three power patterns with less than 2 percent error. 


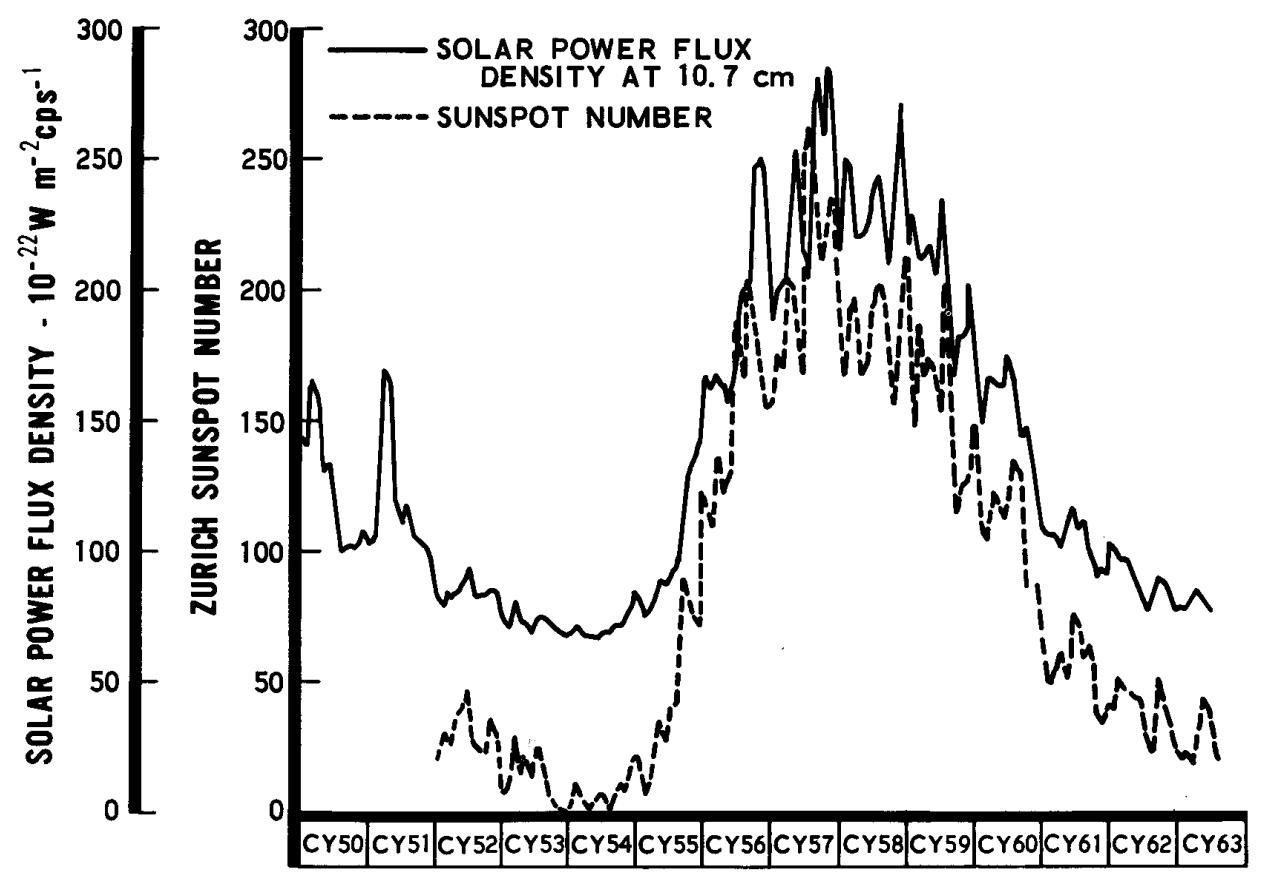

Figure 1. The Slowly Varying Component of Solar Radio Emmission at $10.7 \mathrm{~cm}$ From the Disturbed Sun (NRC, Ottawa) Compared with Zurich Numbers

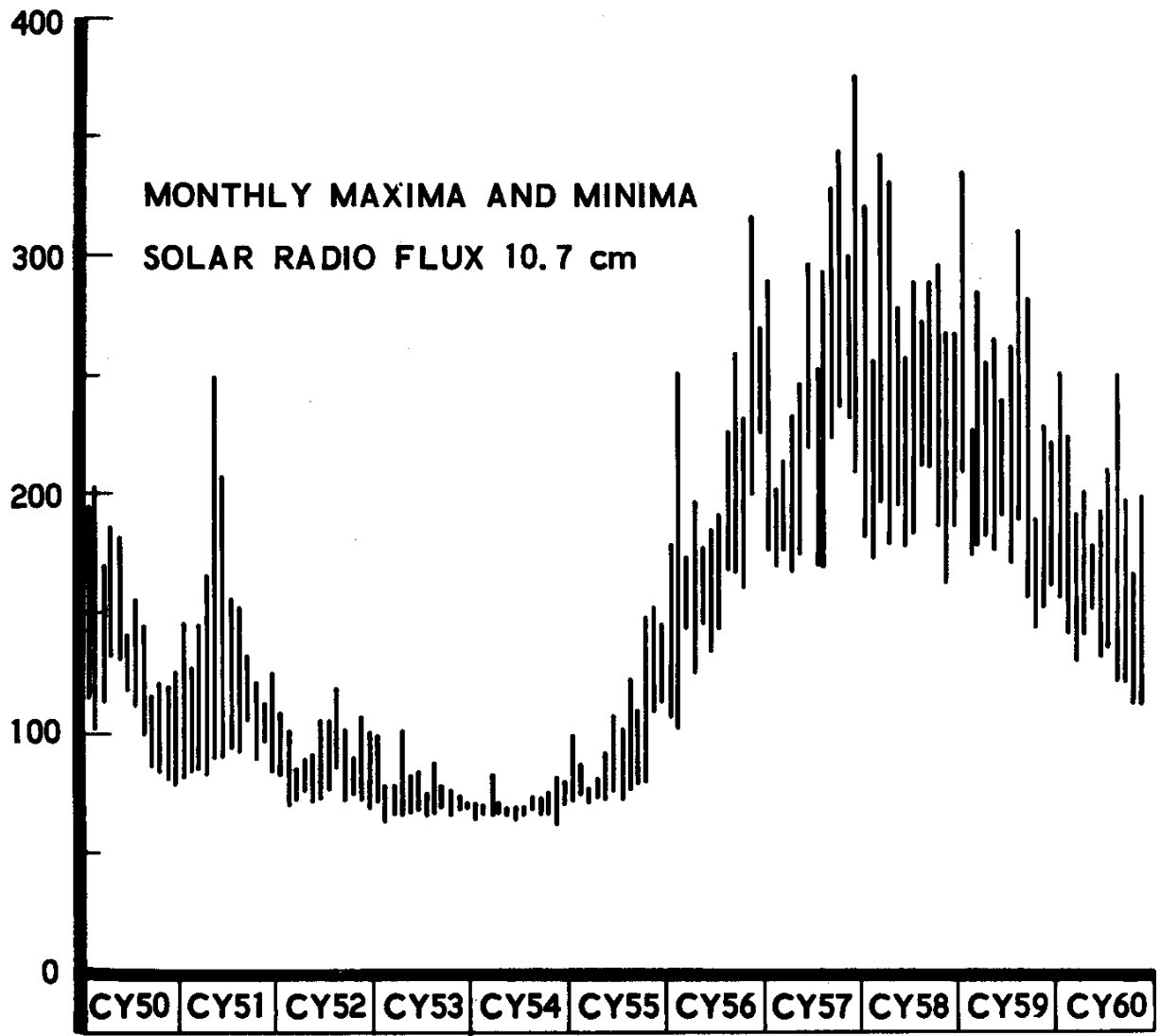

Figure 2. Plot of Monthly High and Low of 10.7-Centimetre Solar Flux. (Units are $10^{-22}$ watts/metre $2 /$ c./s.) (After Covington, 1960) 

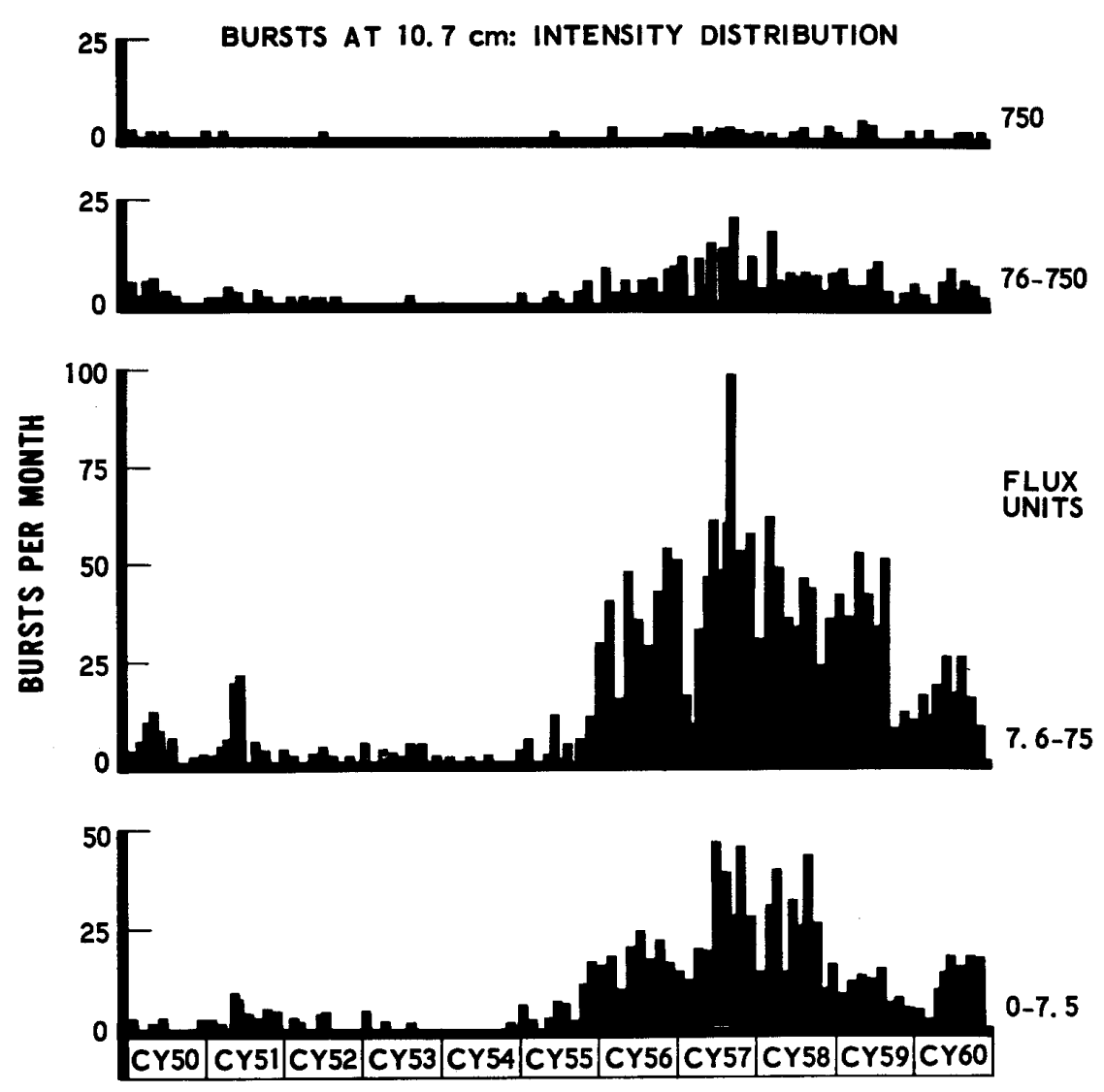

Figure 3. Plots of Number of Bursts Observed in Four Intensity Classes. (Units are $10^{-22}$ watts/metre ${ }^{2} / \mathrm{c} . / \mathrm{s}$.) (After Covington, 1960)

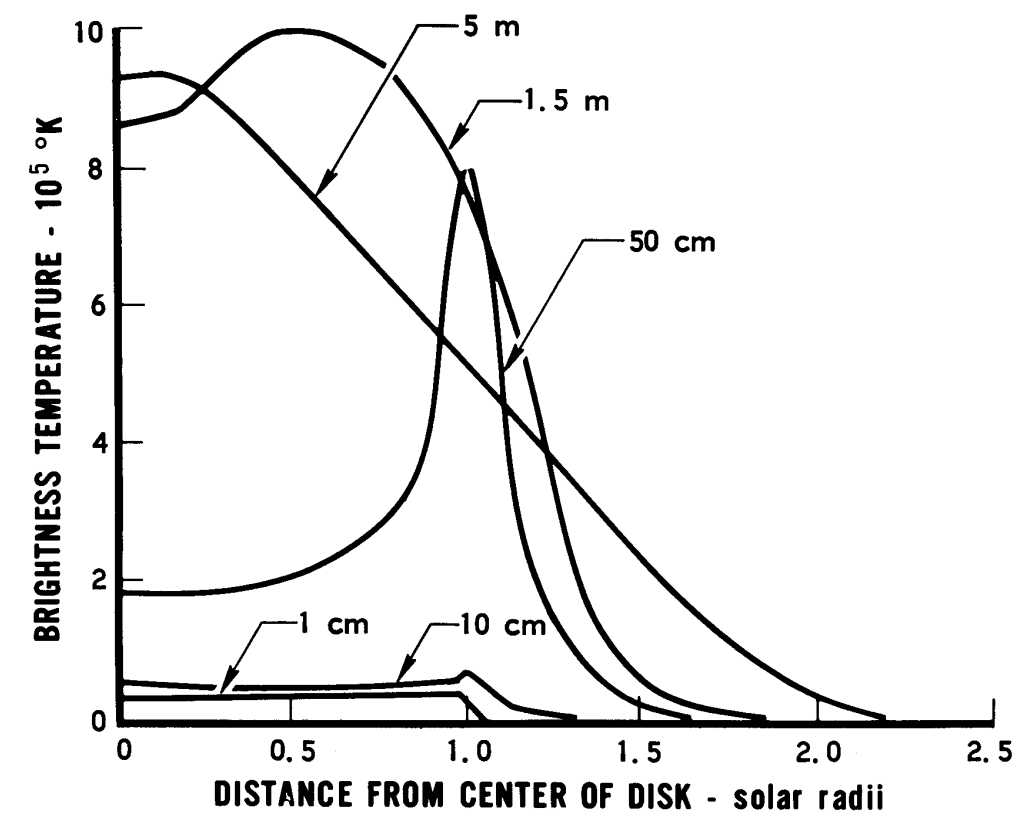

Figure 4. Calculated Distribution of Brightness Temperatures Across the Solar Disk. The Solar Radius is that of the Optical Disk. (After Smerd [Ref. 7]) 


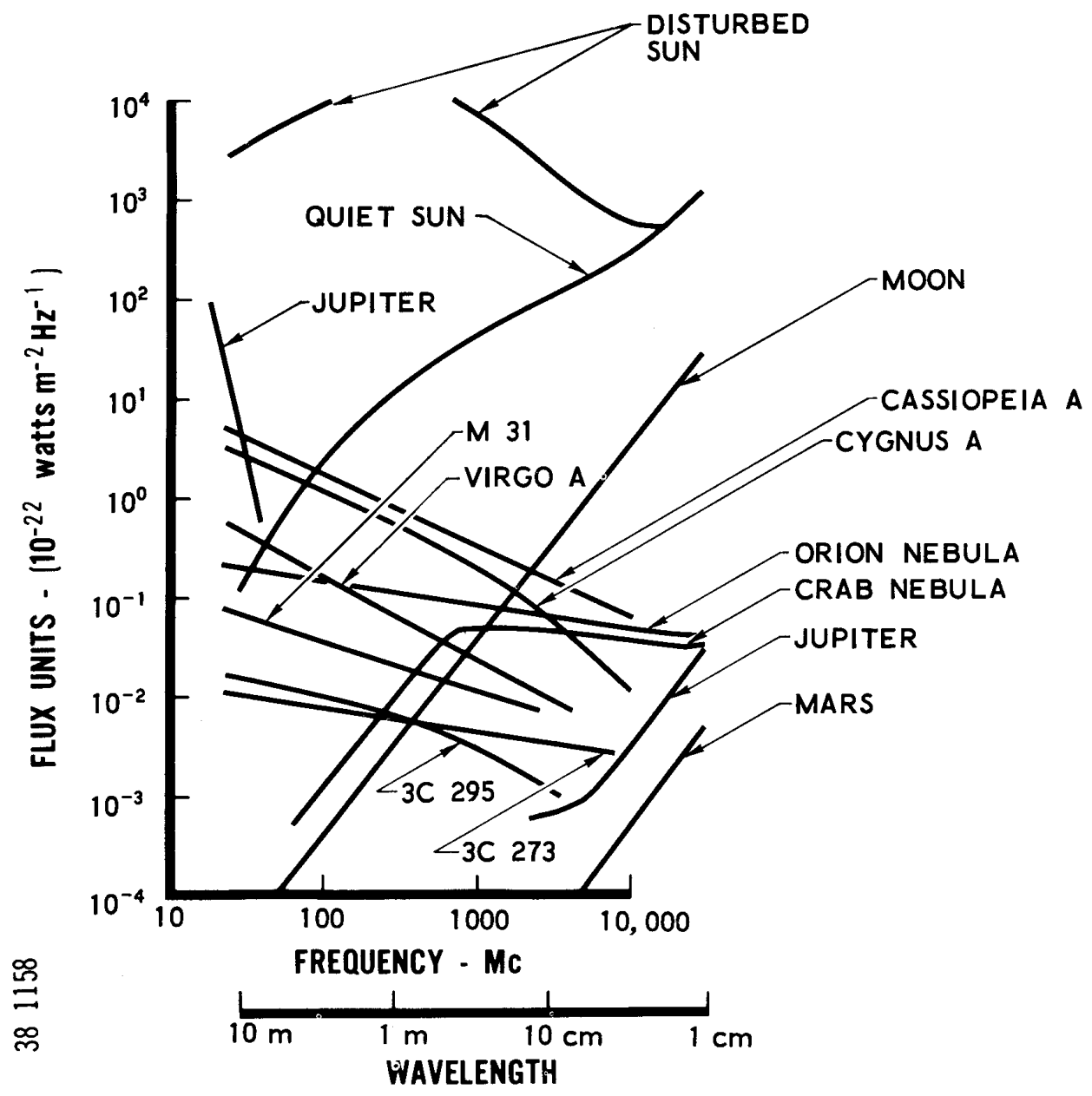

(U) Figure 5. Spectra of Typical Radio Sources (Ref. 10) 